Selected papers from the International Scientific and Practical Conference "Basic Science for Practical Medicine - 2021", 15-18 September 2021, Elbrus v., Russia, http://uniid.kbsu.ru/medicine2021

Original article

\title{
Effectiveness of using fermented herbal preparation in complex therapy of periodontitis developed as an orthodontic treatment complication
}

\author{
Aslan Kh. Sheregov ${ }^{1}$, Zaira F. Kharaeva ${ }^{1}$, Magomet Sh. Mustafaev ${ }^{1}$, Lyudmila G. Korkina ${ }^{2}$, \\ Fatimat R. Batyrbekova ${ }^{1}$, Tauzhan Kh. Agnokova ${ }^{1}$ \\ ${ }^{1}$ Kabardino-Balkarian State University, Nalchik, Russia \\ ${ }^{2}$ Center for Innovative Biotechnological Research Nanolab LLC, Moscow, Russia
}

Received 26 October 2021, Revised 26 November 2021, Accepted 6 December 2021

C 2021, Russian Open Medical Journal

Abstract: Objective - to study the effectiveness of topical application of standardized fermented papaya gel (SFPG) in the complex therapy of moderate chronic periodontitis developed as an orthodontic treatment complication.

Material and Methods - Our study involved 50 patients with periodontitis of a moderate severity developed against the background of a treatment with a fixed orthodontic appliance. Periodontitis has developed as a long-term consequence of the fixed orthodontic appliance. Orthodontic treatment of patients was carried out with metal ligature braces. The patients were divided into two groups: Group 1 with conventional periodontitis treatment supplemented by SFPG applications on the gums for 15 minutes daily during 14 days; Group 2 with conventional treatment alone. The concentration of proinflammatory and anti-inflammatory cytokines in periodontal pockets was determined using solid-phase type of enzyme immunoassay (Vector-Best test system, Russia). The nitrite/nitrate content in the periodontal pocket fluid was determined spectrophotometrically using the Griess reagent kit, following the manufacturer's instructions (Sigma-Aldrich, USA).

Results - Patients receiving SFGP exhibited a reduction in inflammation, manifested in decreased gum bleeding, swelling, hyperemia, and exudate from periodontal pockets. We observed the restoration of the balance of proinflammatory cytokines (IL-1 $\beta$, IL-6) and antiinflammatory interleukin (IL-10), and reduction in the nitrite/nitrate content.

Conclusion - Clinical effectiveness of the therapy with natural fermented herbal preparations may be associated with its antioxidant and antimicrobial properties.

Keywords: periodontitis, orthodontic treatment, interleukins, fermented herbal preparations.

Cite as Sheregov AKh, Kharaeva ZF, Mustafaev MSh, Korkina LG, Batyrbekova FR, Agnokova TKh. Effectiveness of using fermented herbal preparation in complex therapy of periodontitis developed as an orthodontic treatment complication. Russian Open Medical Journal $2021 ; 10$ : e0425.

Correspondence to Aslan Kh. Sheregov. Address: 8 Temrokov St., Village of Hamidiye, Tersky District, Kabardino-Balkarian Republic, Russia 361213. Phone: +79674242560. E-mail: sheregov.aslan@yandex.ru

\section{Introduction}

The prevalence of dental anomalies, according to different authors, ranks third among dental diseases - after dental caries and periodontal diseases [1-4]. It is determined that over $80 \%$ of the population have such anomalies, and $37 \%$ of them require orthodontic care [5]. At the same time, the main method of orthodontic treatment in adults is instrumental - with fixed appliances. It is known that orthodontic treatment deteriorates the hygienic condition of the dentition, and adversely affects the microcirculation in periodontal tissues [6]. Deterioration of the hygienic condition is caused, among other things, by the presence of multiple retention elements on orthodontic appliances [6, 7]. As a result of impaired hygiene, qualitative and quantitative changes occur in the oral microbiome, leading to the development of dysbiosis [7].
It is well known that, in the pathogenesis of gingivitis and periodontitis, the main role is assigned to two factors: the periodontopathogenic microflora and the immunological reaction of periodontal tissues, which has significant autonomy and, in turn, is closely related to the immunity of the entire organism [8, 9].

Orthodontic treatment in patients with inflammatory periodontal diseases (IPD) is among the most complex and urgent problems in dentistry. In recent years, published sources indicated that it is possible to conduct periodontal therapy simultaneously with orthodontic treatment. However, it should be pointed out that is not only possible but quite necessary, since it is preventive measure for the progression of inflammatory reaction in periodontal tissues $[10,11]$. At the same time, it is known that orthodontic forces cause many rearrangements in periodontal 
tissues, which leads, on one hand, to a decrease in their resistance, and, on the other hand, to the activation of bone resorption processes, accompanied by resorption of the bone tissue in jaws and roots of the teeth with the destruction of the periodontal ligament. Currently, there is practically no information in the literature about the methods of preventing such complications; hence, all recommendations are limited solely to the need of regulating the force when moving teeth with the suggestion not to exert too much effort [10-12].

At the same time, the traditional principles of IPD prevention and treatment in patients undergoing orthodontic therapy are not sufficiently effective. Thus, the search for new methods to improve the effectiveness and quality of treatment affecting all links in the IPD pathogenesis in patients undergoing orthodontic treatment is an urgent task today $[11,12]$.

In the ethnoscience of Southeast Asia, various preparations based on papaya are traditionally used as antimicrobial, antiinflammatory and wound healing agents. Standardized fermented preparations based on papaya, as demonstrated in some studies, have effective antimicrobial, immunomodulatory, antioxidant, anti-inflammatory and regenerative effects in both in vitro and in vivo experiments $[13,14]$. In our clinical and laboratory study, we used standardized fermented papaya gel (SFPG) (NBS Co., Ltd., Japan) for topical application in patients with moderate periodontitis developed as a complication of orthodontic treatment.

Objective: to study the effectiveness of SFPG topical use in the complex therapy of chronic periodontitis of moderate severity developed as a complication of an orthodontic treatment.

\section{Material and Methods \\ Patients}

We examined 50 patients (18 men and 32 women) $29-43$ years of age with moderate periodontitis developed as a complication of orthodontic treatment with fixed appliance. The patients underwent dental treatment in private and public clinics of Kabardino-Balkarian Republic (city of Nalchik). Periodontitis of moderate severity developed over the long-term period (15-17 months) after the installation of orthodontic appliance. Orthodontic treatment of patients was carried out with metal ligature braces.

\section{In vivo}

Dental examination of each patient before orthodontic treatment included all of the following: collection of complaints, anamnesis, examination, investigation of the causes of dental anomalies (X-ray examination: orthopantomography, projectional radiography, based on indications of computed tomography of the facial skeleton), the study of diagnostic models, finding out the bad habits of the patient, etc. Before fixing the orthodontic appliance, the patients were given professional hygiene and familiarized with the rules of oral care. In addition to the basic oral hygiene products (toothbrushes and toothpastes), we recommended individually selected additional hygiene products (orthodontic Monopuck toothbrushes, dental floss, dental rinse/mouthwash, etc.).

The patients were divided into 2 groups: Group 1 was experimental (conventional treatment of periodontitis + SFPG), Group 2 was control (conventional treatment alone) (Table 1).
Patients with severe chronic and/or infectious diseases in the acute phase, as well as patients with viral hepatitis, were excluded from the study.

During the simultaneous treatment of patients by a periodontist, orthodontic treatment was suspended for periodontal indications. The conventional treatment of periodontitis included training in oral hygiene, conducting the professional hygiene (removal of supra - and subgingival dental plaque, polishing the surface of the teeth). Based on the indications, proper surgical treatment (curettage) was performed. Topical medicamentous anti-inflammatory treatment included copious rinsing of periodontal pockets with $0.06 \%$ chlorhexidine digluconate solution. In the postoperative period, nonsteroidal anti-inflammatory drugs, desensitizing, and antibacterial therapies were prescribed.

Patients of the experimental group additionally received SFPG in the form of applications to the gum during 15 minutes daily for 14 days (7g of gel per patient per day), which was subsequently washed off with saline, and a recommendation was given to avoid eating during 2 hours after the procedure. The repeated examination was carried out in dynamics - on days 7,14 , and 45 days from the onset of the study.

The comparison group consisted of 13 virtually healthy donors (5 men and 8 women $28-45$ years of age), who were undergoing orthodontic treatment with a fixed appliance and who had their oral cavity sanitized.

The topical objective status of patients with chronic periodontitis was determined by the periodontal index of the World Health Organization (WHO) - community periodontal index of treatment needs (CPITN) and the Silness-Loe Plaque Index. To evaluate the dynamics of the inflammatory process, the papillary marginal alveolar index (PMA) was used in the modification of $S$. Parma (1960). To assess the degree of bleeding, we used the Mühlemann sulcus bleeding index in Cowell's modification.

CPITN determines the need for therapy of periodontal pathologies of any type. Using the special graduated buttonshaped periodontal probe, the condition of periodontal tissues in the area of six teeth was assessed and expressed in points: 0 for no signs of disease; 1 for bleeding after probing and slightly inflamed gingival margin; 2 for supra- and subgingival plaque and gingival sulcus up to $3 \mathrm{~mm} ; 3$ for pathological periodontal pocket of 4-5 mm; 4 for pathological periodontal pocket of 6 or more $\mathrm{mm}$. The final score was calculated from the sum of points divided by 6 . The clinical significance of the CPITN is as follows: 0 means that therapy is not required; 1 signifies training in individual oral hygiene and monitoring of the hygienic condition is necessary; $2-3$ stand for the necessity of professional oral hygiene and training in individual oral hygiene; the score of 4 implies the need for the complex therapy of periodontal tissues [15].

Table 1. Group-based demographic structure of patients with periodontitis developed as a complication of orthodontic treatment

\begin{tabular}{lcccc}
\hline Groups & \multirow{2}{*}{ Number of patients } & Age, years & \multicolumn{2}{c}{ Gender } \\
\hline 1 - experimental & 35 & $29-43$ & 12 & 23 \\
2 - control & 15 & $30-42$ & 6 & 9 \\
\hline
\end{tabular}


Table 2. Dynamics of periodontal condition indices in studied groups during the treatment

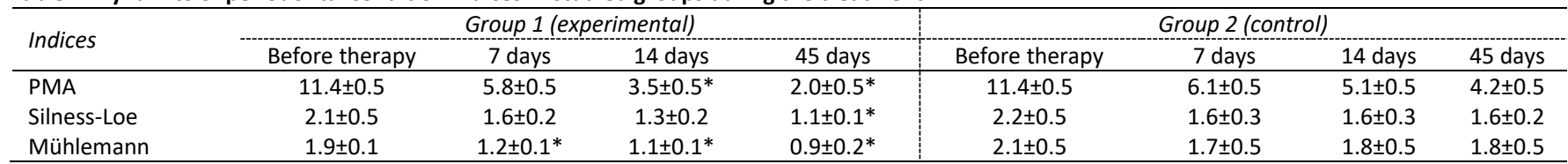

* Statistical significance of differences $p<0.005$, as compared with the values in the comparison group.

Table 3. Topical interleukin status of patients with moderate periodontitis developed as a complication of orthodontic treatment, (pg/mL)

\begin{tabular}{|c|c|c|c|c|c|c|c|c|c|}
\hline \multirow{2}{*}{ Cytokine } & \multirow{2}{*}{ Donor values } & \multicolumn{4}{|c|}{ Group 1 (experimental) } & \multicolumn{4}{|c|}{ Group 2 (control) } \\
\hline & & Before therapy & 7 days & 14 days & 45 days & Before therapy & 7 days & 14 days & 45 days \\
\hline $\mathrm{IL}-1 \beta$ & $8.0 \pm 2.0$ & $34.0 \pm 7.0^{2}$ & $25.0 \pm 5.0^{2}$ & $15.0 \pm 3.0^{1,2}$ & $11.0 \pm 3.0^{1}$ & $37.0 \pm 6.0^{2}$ & $29.0 \pm 5.0^{2}$ & $26.0 \pm 2.0^{1,2}$ & $19.0 \pm 1.0^{1,2}$ \\
\hline IL-6 & $6.0 \pm 1.0$ & $18.0 \pm 3.0^{2}$ & $16.0 \pm 2.0^{2}$ & $14.0 \pm 2.0^{2}$ & $7.0 \pm 2.0^{1}$ & $19.0 \pm 4.0^{2}$ & $17.0 \pm 3.0^{2}$ & $15.0 \pm 2.0^{2}$ & $12.0 \pm 2.0^{1,2}$ \\
\hline
\end{tabular}

${ }^{1}$ Statistical significance of differences $\mathrm{p}<0.005$, as compared with the values in the comparison group; ${ }^{2}$ statistical significance of differences $p<0.005$, as compared with the values in the donors.

Table 4. Dynamics of nitrite/nitrate concentrations in periodontal pockets over the treatment period, $(\mu \mathrm{M} / \mathrm{mL})$

\begin{tabular}{|c|c|c|c|c|c|c|c|c|c|}
\hline \multirow{2}{*}{ Biological material } & \multirow{2}{*}{ Donor values } & \multicolumn{4}{|c|}{ Group 1 (experimental) } & \multicolumn{4}{|c|}{ Group 2 (control) } \\
\hline & & Before therapy & 7 days & 14 days & 45 days & Before therapy & 7 days & 14 days & 45 days \\
\hline Contents of periodontal pockets & $17.0 \pm 1.0$ & $47.5 \pm 5.0^{2}$ & $35.0 \pm 3.0^{2}$ & $29.0 \pm 2.0^{1,2}$ & $20.0 \pm 3.0^{1}$ & $46.5 \pm 5.0^{2}$ & $40.0 \pm 3.0^{2}$ & $36.0 \pm 2.0^{1,2}$ & $34.0 \pm 3.0^{1,2}$ \\
\hline
\end{tabular}

${ }^{1}$ Statistical significance of differences $p<0.005$, as compared with the values in the comparison group; ${ }^{2}$ statistical significance of differences $p<0.005$, as compared with the values in the donors.

The Silness-Loe Plaque Index (1967) takes into account the thickness of the plaque in the gingival region on 4 sections of the tooth surface: vestibular, lingual, distal and mesial. After drying the enamel, the tip of the probe is carried out on its surface at the gingival sulcus. If a soft substance does not stick to the tip of the probe, the plaque index on the tooth area is evaluated as 0 . If the plaque is not visually detected but becomes visible after the probe moves, the index value is 1 . Thin to moderate layer of plaque, visible to the naked eye, is estimated as 2 . Intensive deposition of dental plaque in the gingival sulcus and interdental space is evaluated as 3 [15]. For each tooth, the index is calculated by dividing the sum of the points of four surfaces by 4 . The total index value equals the sum of the values for all examined teeth divided by their number.

The PMA index allows assessing the condition of gums and diagnose the gingivitis. The gums were stained with a SchillerPisarev solution and evaluated by analyzing the degree of dye penetration into the gum: 0 for none and no inflammation; 1 for moderate inflammation of the gingival papilla $(P) ; 2$ for inflammation of the marginal gum (M); 3 for inflammation of the alveolar gum (A). The PMA index was expressed in $\%$ and calculated using the formula: $\mathrm{PMA}=\sum$ points $/ 3 \times$ number of teeth $\times 100 \%$. Evaluation criteria of the PMA index: $30 \%$ imply mild gingivitis; $31-60 \%$ suggest moderate gingivitis; $61 \%$ or more indicate severe gingivitis [15].

The Mühlemann-Cowell index determines the degree of bleeding of the gingival sulcus while probing or pressuring the dental papilla. Evaluation scale is as follows: 0, if there is no bleeding after examination; 1 , bleeding appears no earlier than after 30 seconds; 2 , bleeding occurs either immediately after the examination or within 30 seconds; 3, bleeding is noted when eating or brushing teeth [15].

All index values were measured in dynamics - on days 7, 14 and 45 of the clinical trial.

\section{In vitro}

To determine the concentrations of immunocytokines (IL-1 $\beta$, IL-6, IL-10) in periodontal pockets, we employed the solid-phase enzyme immunoassay (Vector-Best test systems, Russia). The fluid in the periodontal pocket was collected using sterile paper pins, which were inserted into the periodontal pocket for 30 seconds, then placed for $3 \mathrm{~min}$ in an Eppendorf tube with $1 \mathrm{~mL}$ of sterile saline solution. The quantitative content of cytokines in the gingival fluid was expressed in $\mathrm{pg} / \mathrm{mL}$ [16].

The quantitative nitrite/nitrate content in the periodontal pocket fluid $\left(\mathrm{NO}_{2-/ 3}\right.$, expressed in $\left.\mu \mathrm{M} / \mathrm{mL}\right)$ was identified spectrophotometrically using the Griess reagent kit, following the manufacturer's instructions (Sigma-Aldrich, USA).

\section{Statistical analysis}

Statistical processing of clinical and laboratory data was carried out using the STATISTICA 6.0 program (StatSoft, Inc., Tulsa, OK, USA). We checked the data for normality using the ShapiroWilk test. The nonparametric Mann-Whitney $U$ test was employed to compare independent samples between donors and patients with periodontitis. The differences were considered statistically significant at $p<0.05$.

The design of our study was approved by the Ethics Committee at Kabardino-Balkarian State University. The entry of patient data for the analysis was carried out anonymously, with each patient assigned an individual number. The study was conducted in full compliance with the Federal Law of the Russian Federation of December 30, 2017, based on the Declaration of Helsinki (with its later amendments).

\section{Results}

When examined by a dentist before the use of SFPG, the studied patients of both groups with moderate periodontitis, developed as a complication of orthodontic treatment, had 
persistent clinical signs of periodontal tissue inflammation, which was confirmed by a statistically significantly increased average CPITN value of 1.9 (its normal range is between 0 and 1 ). At the same time, in 37 patients ( 13 men and 24 women) of both groups, bleeding, swelling, itching in the gum area, increased sensitivity of teeth to temperature and chemical influences, augmented mobility of teeth, unpleasant odor from the oral cavity (halitosis) prevailed among the symptoms of gum inflammation. Remaining 13 patients ( 5 men and 8 women), in addition to the above complaints, noted other signs of general body intoxication: weakness and malaise.

The topical use of SFPG in Group 1 was well tolerated by patients, among whom no adverse effects were registered throughout the study (no allergic reactions or other signs of intolerance).

Correction of individual oral hygiene, accompanied by professional hygiene, significantly improved the condition of the oral cavity in all examined patients. Such clinical signs as itching, bleeding of the gums when eating and brushing teeth, swelling and hyperemia in the periodontal pockets, decreased. However, the clinical efficacy was evidence-based because of the presence of a statistical difference $(p<0.005)$ between the groups of patients (Table 2). The Mulleman index from day 7 of observation was significantly lower in Group 1 (where SFPG was used) and remained such in the long run - on days 14 and $45(p<0.005)$ (Table 2). Statistical significance of differences in the PMA index values between the groups emerged on day 14, whereas the Silness-Loe index normalized in the experimental group of patients by day 45 after the onset of therapy (Table 2 ).

When determining the concentrations of two proinflammatory cytokines (IL-1 $\beta$, IL-6) and anti-inflammatory cytokine, IL-10, in the periodontal pockets of patients with moderate periodontitis, developed as a result of an orthodontic treatment, we discovered that prior to the study, the content of IL-1B, IL- 6 in both groups was statistically significantly higher, and IL-10 concentration was significantly lower, compared with those of healthy donors $(p<0.005)$ (Table 3). In the course of the therapy, cytokine concentrations tended to normalize, reaching normal levels by day 45 in patients of the experimental group receiving SFGP. However, in the control group, normalization of the cytokine levels in periodontal pockets was not registered at all; hence, the intergroup statistically significant difference in IL-1 $\beta$ concentration was detected on day 14 (Table 3).

The nitrite/nitrate content in the periodontal pocket fluid was significantly increased before the onset of therapy in both groups of patients, as compared with the values of healthy donors $(p<0.005)$ (Table 4). A statistically significant difference in nitrite/nitrate content between the groups was detected on day 14 of the study. The values as close as possible to those of healthy donors were registered by day 45 in the experimental group receiving SFPG topical applications (Table 4).

\section{Discussion}

An orthodontist often confronts the situations where, due to a pronounced inflammatory reaction of the periodontal tissues, it is necessary to stop orthodontic treatment in order to carry out periodontal treatment, and only then to resume bite correction, or resort to a decrease in orthodontic forces in used appliances, which lengthens the treatment period. At the same time, careful individual hygiene, along with periodically conducted professional hygiene, do not lead to the desired effect on the oral microflora activity in patients with periodontitis. In such cases, the periodontist is forced to prescribe the patient a course of antiseptic treatment of the oral cavity. As a rule, such procedures are assigned without taking into account the sensitivity of microflora to antiseptics, which provokes the development of dysbiosis in the oral cavity. Besides, such antimicrobial effect is carried out against the background of a decrease in local immunity and changes in the protection of periodontal tissues, which aggravates the patient's condition $[14,17]$.

Chronic local inflammation in periodontitis was confirmed by a significantly increased initial level of main proinflammatory cytokines (IL-1 $\beta, \mathrm{IL}-6)$ and a reduced level of the anti-inflammatory cytokine IL-10, which, in turn, is also a reflection of the reaction of phagocytic cells to periodontal tissue trauma and restructuring of periodontal structures by forced displacements of teeth under the influence of the traction force of braces for a longer time [18]. The therapeutic course with the use of SFGP led to a complete normalization of the mediator parameters of the periodontal tissues in patients.

The results of earlier clinical and laboratory studies and the assessment of the mediator features of periodontal tissues in patients with moderate periodontitis, developed against the background of orthodontic treatment, showed that at later stages of orthodontic treatment, with the progression of periodontitis, a complex of persistent mutually supportive changes in periodontal tissues at different levels of pathogenesis is revealed in patients [19]:

i) An increased concentration of periodontopathogens in the gingival pockets, which is associated, among other things, with the difficulties of oral hygiene during orthodontic treatment with fixed appliances and favorable conditions for microbial activity in the affected tissues:

ii) An innate immune cell activation produces proinflammatory cytokines (IL-1 $\beta$, IL-8) in periodontal tissue, which, in turn, determines the severity of destructive processes in the gingival epithelium and bone tissue. Presumably, IL-1 13 , a powerful stimulator of bone resorption, is involved in the pathogenesis of periodontal tissue destruction [19];

iii) Depletion of antioxidant activity in periodontal tissues, against the background of prolonged hyperproduction of reactive oxygen/nitrogen species.

The obtained data indicate that the pathogenetic parameters and changes in periodontal tissues in patients with periodontitis, caused by orthodontic treatment, are virtually identical to those in periodontitis of a different etiology. In this regard, it is necessary to develop a pathogenetically justified comprehensive prevention of complications for this group of patients.

The preparation based on fermented papaya has antioxidant properties and antimicrobial effects due to the inhibition of protective enzymes in bacteria $[13,14,20]$. Topical application of the drug in the form of applications allowed reducing the inflammation of periodontal tissues via restoring the pro-oxidantantioxidant balance and inhibiting the accumulation of microbes in periodontal pockets, which led gradually to an improvement in the 
trophic tissues. Clinical efficacy correlated with a decrease in laboratory indicators of periodontal inflammation.

\section{Conclusion}

Our clinical and laboratory studies, involving the topical application of SFPG in the complex treatment of periodontitis that developed against the background of orthodontic treatment, showed a positive trend persisting throughout the entire period of observation. Favorable clinical results were associated with the anti-inflammatory effect of the herbal preparation application manifested via normalization of the cytokine status and the freeradical status of tissues.

A comparative assessment of the topical SFGP application outcomes in treating chronic generalized periodontitis that developed against the background of orthodontic treatment showed that the therapeutic effectiveness of the method is substantially superior to conventional treatment methods. That is why we strongly recommend a topical application of this phytopreparation, based on fermented plant material, for the treatment of inflammatory periodontal diseases.

\section{Funding}

The study was performed within the framework of the Public Procurement No. 0669-2020-0008 of the Ministry of Science and Higher Education of the Russian Federation.

\section{Conflict of interest}

The authors declare no conflicts of interest.

\section{References}

1. Alimsky AV, Dolgoarshinnykh AYa. Study of age dynamics of dentoskeletal anomaly frequency among children population. Orthodontics 2007; (2): 10-12. Russian https://www.elibrary.ru/item.asp?id=12845117.

2. Danilova MA, Tsarkova OA, Ponomareva ML. The structural analysis of risk's factors of dentoalveolar anomalies at children of preschool age. Pediatric dentistry and dental profilaxis 2014; 13(2(49)): 15-17. Russian. https://www.elibrary.ru/item.asp?id=22263924.

3. Kuzmina EM, Kuzmina IN, Smirnova TA. Oral diseases prevalence among russian population. Teeth condition. Dentofacial abnormalities. Prosthetic treatment need. Moscow: Moscow State University of Dentistry. 2009; $236 \quad$ p. https://www.elibrary.ru/item.asp?id=21357111.

4. European Orthodontic Health Insurances. Karlbergsv: Committee of European Health Insurances. 2010; 22 p.

5. Gunenkova IV, Pogosyan NS, Gurevich KG, Fabrikant EG. Indicators of quality of life in orthodontic treatment with a fixed appliance. Orthodontics 2008; (1): 8-10. Russian. https://www.elibrary.ru/item.asp?id=10439861.

6. Kharaeva ZF, Blieva LZ, Sheregov AKh, Barokova EB, Shogenova IZ, Khulayeva $\mathrm{Al}$, et al. Comparative analysis of adhesive properties of ceramic and metal bracket systems. Clinical Dentistry (Russia) 2019; 2(90): 42-44. Russian. https://doi.org/10.37988/1811$153 \times 2019242$.

7. Sevbitov AV, Nevdakh AS, Platonova VV. A new approach to the treatment traumatogenic erosive-ulcerative lesions of the oral mucosa at orthodontics patients. Periodontology (Russia) 2016; 21(3(80)): 1214. Russian. https://www.elibrary.ru/item.asp?id=27185775.

8. Melekhov SV, Kolesnikova NV, Ovcharenko EU. State of local immunity and microbiocenose oral cavities at patients with chronic generalized periodontitis. Periodontology (Russia) 2013; 18 1(66): 3-9. Russian. https://www.elibrary.ru/item.asp?id=19048540.

9. Ivanyushko TP, Ter-Asaturov GP, Budanova EV, Khoroshilova NV. Features of the oral microflora and local immunity factors in patients with chronic periodontitis. Stomatology 2000; (3): 26-27. Russian.

10. Kuatbaeva UA. Risk factors of periodontal disease in individuals with a bracket system (according to literature). Vestnik KazNMU 2017; (3): 179-184. Russian. https://cyberleninka.ru/article/n/faktory-riskazabolevaniy-parodonta-u-lits-s-breket-sistemami-po-dannymliteratury.

11. Arsenina OI, Grigoryan AS, Frolova OA, Petrunina OV. Diagnosis and treatment of inflammatory processes in the periodontium occurring during orthodontic treatment. The Dental Institute 2005; (1(26)): 50 55. Russian. https://www.elibrary.ru/item.asp?id=15267369.

12. Blashkova SL, Mustafin IG, Khaliullina GR. Features of the processes of immune regulation in periodontal tissues in patients undergoing orthodontic treatment. Periodontology (Russia) 2016; 21 (3(80)): 2326. Russian. https://www.elibrary.ru/item.asp?id=27185778.

13. Osato JA, Korkina LG, Santiago LA, Afanas'ev IB. Effects of bionormalizer (a food supplementation) on free radical production by human blood neutrophils, erythrocytes, and rat peritoneal macrophages. Nutrition 1995; 11 (5 Suppl): 568-572. Russian. https://pubmed.ncbi.nlm.nih.gov/8748224.

14. Mikhalchik EV, Ivanova AV, Anurov MV, Titkova SM, Penkov LYu, Kharaeva ZF, et al. Wound-healing effect of papaya-based preparation in experimental thermal trauma. Bulletin of Experimental Biology and Medicine 2004; 137: 560-562. Russian. https://doi.org/10.1023/B:BEBM.0000042711.31775.f7.

15. Molokov VD, Dorjieva ZV, Bivalceva SU. Methodological Manual for Self-Training of Students at School of Dentistry on the topic, Index Assessment of Dental Caries and Periodontal Diseases. Irkutsk: Irkutsk State Medical University, 2008; 23 p. Russian. https://mir.ismu.baikal.ru/src/downloads/eb639cc3 indeksnaya otse nka kariesa_i zabolevaniiy_parodonta.pdf.

16. Balikin RA. Evaluation of personalized immunotherapy efficiency in surgical treatment periodontitis. PhD dissertation. Moscow. 2019; 118 p. Russian. https://www.cniis.ru/downloads/dis/dis_Balykin.pdf.

17. Shimansky ShL, Chilikin VN, Rumyantsev VA, Yesayan LK. Evaluating the role of periodontal pathogenic microflora of the oral cavity in the development of chronic obstructive pulmonary disease. Medicine. Science and Education 2017; (22): 101-104. Russian.

18. Shimanskii ShL, Chilikin VN, Malyshev Ilu, Suvorova IA, Rumiantsev VA, Denis AG. Phagocytes protection of periodontium and its ways activation. Stomatology 2013; 92(5): 64-69. Russian. https://elibrary.ru/item.asp?id=21084672.

19. Sheregov $\mathrm{AH}$. Immunological status of gingival tissue in patients with periodontal diseases of varying severity undergoing orthodontic treatment. In: Proceedings of the International Scientific Conference of Undergraduate and Graduate Students, and Young Scientists, Innovations in Fundamental and Clinical Medicine 2020: 420-426. Russian.

20. Badalian ZV, Makarova LM, Pogorely VE, Temirbulatova AM, Stepanova EF. Comparative pharmacological study of the fermented juice of plantain. Belgorod State University Scientific bulletin. Medicine. Pharmacy 2012; (10-3(129)): 121-123. Russian. https://elibrary.ru/item.asp?id=20359782.

Authors:

Aslan Kh. Sheregov - Graduate Student, Institute of Dentistry and Maxillofacial Surgery, Kabardino-Balkarian State University, Nalchik, Russia. Zaira F. Kharaeva - DSc, Professor, Chair of the Department of Microbiology, Virology, and Immunology, School of Medicine, KabardinoBalkarian State University, Nalchik, Russia. https://orcid.org/0000-00032302-2491. 
Magomet Sh. Mustafaev - DSc, Professor, Director of the Institute of Dentistry and Maxillofacial Surgery, Kabardino-Balkarian State University, Nalchik, Russia. https://orcid.org/0000-0002-4042-9421.

Lyudmila G. Korkina - DSc, Professor, Director General of the Center for Innovative Biotechnological Research Nanolab LLC, Moscow, Russia. https://orcid.org/0000-0003-3887-8866.

Fatimat R. Batyrbekova - PhD, Associate Professor, Institute of Dentistry and Maxillofacial Surgery, Kabardino-Balkarian State University, Nalchik, Russia.

Tauzhan Kh. Agnokova - PhD, Associate Professor of the Institute of Dentistry and Maxillofacial Surgery, Kabardino-Balkarian State University, Nalchik, Russia. 